\title{
An Effective Inpainting Technique for Hole Filling in DIBR Synthesized Images
}

\author{
Richard Ramírez, Fernando Jaureguizar, Narciso García, Julián Cabrera
}

\begin{abstract}
This paper proposes an effective image inpainting method for Depth-Image-Based Rendering (DIBR) synthesized images technique to solve disocclusion holes problems in texture images and their respective depth maps. Disoccluded areas, or holes, are classified depending on the size of the hole, being more critical those that appear in the border of the objects, normally between two great depth value differences. Exemplar-based inpainting contains the essential process required to replicate texture and structure for large dissocluded area. The proposed technique focus on preprocessing to improve gradient detection using sobel algorithm followed by a depth-aided exemplar-based technique gives encouraging results.
\end{abstract}

Keywords-view synthesis; DIBR; FTV; inpainting;

\section{INTRODUCTION}

The fast development in 3D technology in recent years, has made the 3D displays an effective way to enjoy 3D content and so popular around the world. Today, there are TV sets, mobile devices and displays with 3D display function available in the market; hence, there are more technical demands in the 3D field. 3D image usually contains information of two or more viewing angles from left and right eyes that create a stereo feeling to both eyes while people are viewing the screen [1]. Autostereoscopic displays with 2D+Depth format has increased the number of available free viewing positions and one of the reasons is the optimal quality of the synthesized views generated by the specific hardware used for this purpose. Taking this fact as a priority, effective, fast and low computational cost algorithms are necessary.

Autostereoscopic displays avoid the use of especial 3D glasses, as steoroscopic displays do, have been increasing the number of free viewpoints, and nowadays there are displays providing even 28 viewpoints to have more realistic perception, with intentions to increase this number. This technology is able to provide a 3D experience using Video plus Depth format that has the flexibility to warp and render virtual views in specified positions through the Depth-Image Based Rendering (DIBR) using projective geometry applied to the reference view images and their associated depth maps, suitable for autostereoscopic displays based on depth maps from scenes. After the warping process, new background areas appear without corresponding information about the reference view, and, for this reason, many inpainting methods based on exemplar-based techniques has been applied to solve this problem [2][3][4], each one focused on optimization and giving a realistic synthesized view with minimal presence of artifacts.

The problem with the DIBR is the appearance of dissoclusions in the virtual views that reduce the visual quality; these disocclusions look like holes in the synthesized images and could degrade the visual quality of the synthesized images greatly [5]. In this context, it is defined the difference between background and foreground of the scene: the foreground is the area or object closest to the camera in the scene and the background being otherwise.

\section{DEPHT AIDED EXEMPLAR BASED INPAINTING}

Inpainting methods use the structure and texture to fill the holes in an image (I) (Fig. 1). The key steps in these methods are estimating the filling order and finding the best match between the target patch at the boundary $(\partial \Omega)$ of the hole and the source patch in the source region $(\Phi)$. The filling order is determined by the structure (data term) and the amount of reliable information (confidence term). The best source patch $\left(\Psi_{q}\right)$ is found by minimizing the sum of square differences (SSD) as the Criminisi algorithm [4] (Fig. 2). The source region in the inpainting process is the region in the image where the best source patch is found to fill the missing target regions $(\Omega)$. The great advantage of this method is that the patches are created from the image itself and copied them into the hole exploiting the redundancy and avoiding interpolated patches that increase the number of artifacts or additional filter that blurs the image. The order in which this filling process is executed determines the quality of the output image.

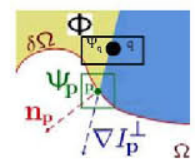

Fig. 1 Given patch $\Psi_{p}, n_{p}$ is the normal to the contour $\partial \Omega$ of the target region $\Omega$ and $\nabla I_{\mathrm{p}}^{\perp}$ is the isophote (direction and intensity) at point $\mathrm{p}$.

Confidence term $C(\mathrm{p})$ and Data term $D(\mathrm{p})$ are defined as follows:

$$
\begin{array}{r}
C(\mathrm{p})=\frac{\sum_{\mathrm{q} \in \Psi_{\mathrm{p} n(I-\Phi)}} C(\mathrm{q})}{\left|\Psi_{\mathrm{p}}\right|} \quad D(\mathrm{p})=\frac{\left|\nabla I_{\mathrm{p}}^{\perp}\right|}{\alpha} \\
\Psi_{\widehat{\mathrm{q}}}=\arg \min d\left(\Psi_{\widehat{\mathrm{p}}}, \Psi_{\mathrm{q}}\right)
\end{array}
$$


where $\left|\Psi_{p}\right|$ is the area in term of pixels of the patch $\Psi_{p}$ and $\alpha$ is a normalization factor (255).

On the other hand, depth-aided exemplar-based techniques use depth map information to increase an accurate priority $P(\mathrm{p})$ and filling order adding a Level regularity term $L(\mathrm{p})$ defined as:

$$
L(\mathrm{p})=\frac{|Z \mathrm{p}|}{|Z \mathrm{p}|+\sum_{\mathrm{q} \in \Psi_{\mathrm{p} \cap \Phi}}(Z \mathrm{p}-\overline{Z \mathrm{p}})^{2}}
$$

where $|Z \mathrm{p}|$ is the area in term of pixels of the patch $Z \mathrm{p}$ and $\overline{Z p}$ the mean value. Finally, the priority is defined as the product of these terms:

$$
P(\mathrm{p})=C(\mathrm{p}) D(\mathrm{p}) L(\mathrm{p})
$$

The proposed method relies on the depth-based inpainting method in [6] (Fig. 3), but employing first an enhanced gradient detection for texture images, based on Sobel, to get an optimal image gradient in order to improve accuracy for data term $D(\mathrm{p})$. Furthermore, the boundary of large disocclusion areas allow the classification of the image in background and foreground depending on their neighbour's depth values (Fig. 4). Due to the fact that a disocclusion area is the lack of background information and the depth map contains only gray values, is easy to fill the warped depth map copying background information from left to right direction with a horizontal filling order (Background-Foreground horizontal filling) avoiding complex and high computational cost.

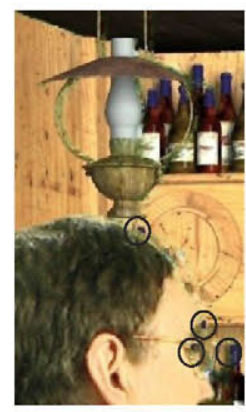

Fig. 2 Criminisi.

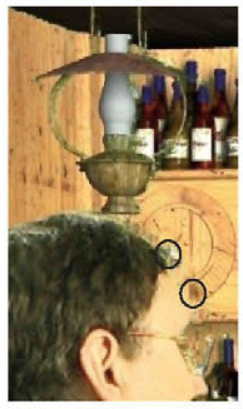

Fig. 3 Depth-aidedexemplar.

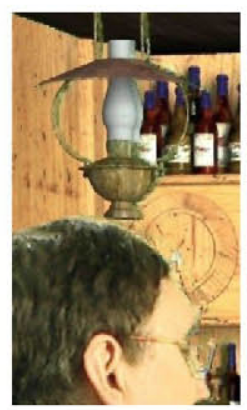

Fig. 4 Proposed optimal gradient.

\section{EXPERIMENTAL RESULTS}

Experimental results show that an efficient and optimal gradient detection plus depth-aided exemplar-based inpainting provides better results in texture image as shown in Fig. 4, but if it is applied to fill the associated depth map, an inconsistent with reality result is obtained (Fig. 5). For this reason, horizontal Background-Foreground horizontal filling technique as shown in Fig. 6 has been used. The tests have been done in the HSV (Hue-Saturation-Value) color space with better results than in the RGB one, encouraging to keep going with the research in this area.

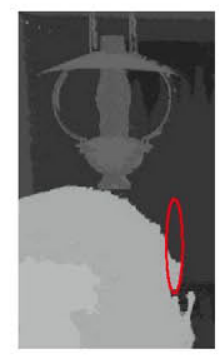

Fig.5 Depth-aided exemplar based inpainting.

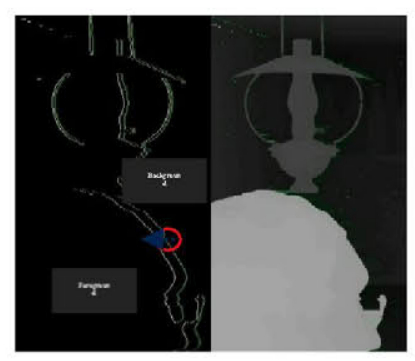

Fig.6 Background-Foreground Horizontal filling technique.
Table 1 shows PSNR and SSIM results in order to analyze the performance of the proposed algorithm against the View Synthesis Reference Software [7] version 2.0 (VSRS 2.0). It can be seen a small improvement in the purpose inpainting method compared with the other ones tested.

TABLE I. RESUlts OF ALGorithMs TESTED AND PROPOSED METHOD

\begin{tabular}{|l|c|c|c|}
\hline \multicolumn{1}{|c|}{ Method } & SSIM(\%) & PSNR(dB) & Time(s) \\
\hline Proposed optimal gradient & 77.0380 & 24.33892 & 70.7153 \\
\hline Depth aided exemplar based & 76.9821 & 24.27826 & 67.8636 \\
\hline Criminisi & 76.9321 & 24.19953 & 62.9795 \\
\hline
\end{tabular}

\section{CONCLUSIONS}

This paper presents an efficient technique to solve large disocclusion filling problems in DIBR virtual views. This technique uses a low complex computational cost algorithm to render virtual views with good performance, suitable for electronics devices looking for fast implementations as is being needed in Free Viewpiont TV (FTV) systems with autostereoscopic displays.

\section{REFERENCES}

[1] F. Cheng and M.Loo. "An Image Inpainting Method for Stereoscopic Images Based on Filling Route," in Proc. of Intelligent Information Hiding and Multimedia Signal Processing (IIH-MSP), 2014 Tenth International Conference on. IEEE, pp. 399-402, 2014.

[2] S.Muddala, M. Sjostrom and R. Olsson, "Depth-based inpainting for disocclusion filling," in proc. Of 3DTV-Conference: The True Vision Capture, Transmission and Display of 3D Video (3DTV-CON), pp. 1-4, 2014.

[3] X. Xuyuan, P. Lai-Man, C. Chun-Ho, F. Litong, H. Ka, C. Kwok-Wai, "Depth-aided exemplar-based hole filling for DIBR view synthesis," (ISCAS), IEEE International Symposium on Circuits and Systems Circuits and Systems, pp. 2840 - 2843, 2013.

[4] A.Criminisi, P. Perez and K. Toyama, "Region filling and object removal by exemplar-based image inpainting," IEEE Trans. Image Process, vol. 13, no. 9, pp. 1200-1212, 2004.

[5] C. Kun-Ming, L. Ting-Ching and H. Yueh-Min, "Parallax Guided Disocclusion Inpainting for 3D View Synthesis," IEEE International Conference on Consumer Electronics (ICCE) , pp. 398-399, 2012.

[6] I. Daribo and H. Saito, "A novel inpainting-based layered depth video for 3dtv," IEEE Transactions on Broadcasting, vol. 57, no. 2, pp. 533$541,2011$.

[7] M. Tanimoto, T. Fujii, and K. Suzuki, "View synthesis algorithm in view synthesis reference software 2.0 (VSRS2.0)," Lausanne, Switzerland, ISO/IEC JTC1/SC29/WG11 Doc. M16090, Feb. 2009. 